\title{
A Complete 8-GHz QPSK-MODEM Featuring Novel Subcarrier and Data Synchronization for Optical Communications
}

\author{
Pandelis Kourtessis, Member, IEEE, and Stuart D. Walker
}

\begin{abstract}
We describe the application of subcarrier multiplexing (SCM) to enable broadband transmission beyond the modal bandwidth of multimode fiber (MMF) optical links. The application of a quadrature phase-shift keying modem featuring novel carrier and symbol-timing recovery circuits, demonstrates significant enhancement in channel capacity. By using a 2.7-GHz pilot-tone-injected phase-locked loop (PLL) and referencing subscriber data to a prescaled version of the subcarrier, zero-latency carrier and data synchronization has been achieved exhibiting $675 \mathrm{Mb} / \mathrm{s}$ bit-oriented clock extraction in network terminal equipments. The novel scheme is scalable to higher aggregate rates and upgrades to existing installed-base $50 \mu \mathrm{m}$ MMF infrastructure to provide low-cost, high-capacity interconnects for storage area and campus network applications.
\end{abstract}

Index Terms-Analog integrated circuits, modems, optical fiber communication, phase-locked loops (PLLs), quadrature phaseshift keying (QPSK), subcarrier multiplexing (SCM), synchronous detection.

\section{INTRODUCTION}

I NSTALLED-BASED multimode fiber (MMF) links are limited in bandwidth distance products to approximately $500 \mathrm{MHz} / \mathrm{km}$ [1]. Realization of higher transmission rates and lengths require techniques capable of overcoming primarily modal bandwidth limitations [2]. It is well known that basic wavelength-division multiplexing (WDM) techniques and multilevel coding yield improvements in the bandwidth capacity product of MMF links [3], and also that subcarrier multiplexing (SCM) can provide capacity enhancement in single- and multiuser networks [4]. This latter advantage is achieved by using relatively flat regions of the fiber response, outside its nominal modal bandwidth [5].

This experimental work served the purpose of demonstrating a high-frequency quadrature phase-shift keying (QPSK) transmission system with a number of novel features, compared with

Paper approved by C. Tepedelenlioglu, the Editor for Transmission Systems of the IEEE Communications Society. Manuscript received October 3, 2005; revised July 7, 2006 and September 26, 2006. This work was supported by EPSRC under the Low-Cost Subcarrier Optical Networks and PHOTON projects. This paper was presented in part at the Optical Fiber Communications Conference, Anaheim, CA, March 2002, and in part at the European Conference on Optical Communications, Copenhagen, Denmark, September 2002.

P. Kourtessis was with the University of Essex, Colchester CO4 3SQ, U.K. He is now with the University of Hertfordshire, Science and Technology Research Institute, Optical Communication Systems and Networks Group, Herts AL10 9AB, U.K. (e-mail: P.Kourtessis@ herts.ac.uk).

S. D. Walker is with the Optical Systems Research Group, Department of ESE, University of Essex, Colchester CO4 3SQ, U.K. (e-mail: pkourt@ essex.ac. uk).

Digital Object Identifier 10.1109/TCOMM.2007.896048 conventional designs, to modulate the subcarrier frequencies prior to their application over the optical links. SCM was incorporated to take advantage of the potential bandwidth and demonstrate the transmission of broadband signals over frequency ranges much higher than the MMF's modal bandwidth [6].

The employment of SCM to develop high-capacity optical networks provides a number of advantages compared with widely used time-division multiplexing (TDM). It allows the designer to fully exploit the available bandwidth by combining several subcarrier channels, the capacity of each depending upon the system properties. A range of inexpensive, microwave components like double-balanced mixers and combiners are available in order to perform these tasks.

Also the photodiode at the fiber end detects all subcarrier channels over the total system bandwidth, but only the desired channels have to be demodulated using again conventional microwave components. This way, high-speed, expensive multiplexers and demultiplexers are not required, in contrast to TDM [4].

SCM also offers considerable versatility, since it can be implemented both electrically and optically. Several optical sources, each modulated with various subcarrier signals, can be combined in a passive optical network [7]. In local-area networks (LANs), one channel can be transmitted from each laser, whereas in a video distribution network, many subcarriers are sent from a single source. This versatility of SCM provides the freedom to select upon, or combine a number of, different design approaches and implement the most suitable as far as efficiency and cost effectiveness.

QPSK is proposed to modulate the SCM channels, since it demonstrates enhanced transmission capacity compared with its binary counterpart with the same requirement in power for error-free detection and equivalent complexity, as far as subcarrier and clock recovery concerns. It provides a very strong candidate for demonstrating much higher capacity over the available MMF bandwidth than the one achieved with binary (B)PSK modulation [8]. A critical issue taken into consideration during system design was the subcarrier (CR) and symbol-timing recovery (STR) circuits necessary to extract the required signals for demodulation and symbol detection. The main concern was the high frequencies involved that would make conventional CR and STR circuits difficult to realize in practice.

Although researchers have investigated the use of a pilottone-based phase-locked loop (PLL) to achieve carrier and clock extraction with QPSK signaling [9], the proposed design 
suffers from varying frequency shift between the transmitted and recovered subcarrier frequencies, resulting in the PLL losing "lock," due to unstable loop-filter performance and lack of synchronization between the subcarriers and transmitted sequences. In addition, subcarrier extraction in the receiver is performed with the help of a local oscillator and a duplication of the source generator electronics. This is performed in order to emulate the generation of multiple subcarriers in the transmitter, which increase system complexity and cost considerably at $\mathrm{Gb} / \mathrm{s}$ frequency rates. Furthermore, the open-loop nature of such a system design does not allow a compensation of phase error at the phase detector output that could result in clock jitter.

We propose the application of a novel QPSK system featuring zero-latency carrier extraction and data synchronization based on a basic, pilot-tone PLL and "locking" of the transmitted clock to a single subcarrier. Although the demonstrated setup employs quadrature phase modulation, system upgrades to higher level phase modulation can be easily implemented. This is achieved through the addition of an appropriate phase shifter in the transmitter and receiver, and by using the constant phase-shift property and phase margin of the demonstrated PLL to extract all subcarrier phases. The application of a $0.035 \mathrm{~V}$ pilot tone at the transmitted signal spectrum resulted in a maximum power penalty of $0.79 \mathrm{~dB}$ in the received signal signal-to-noise ratio (SNR) to achieve a bit-error rate (BER) of $10^{-9}$, compared with a conventional experimental setup devised for relative system performance evaluation purposes. Thus the use of a pilot tone allows simple $\mathrm{CR}$ extraction at $\mathrm{GHz}$ frequencies with a minimal penalty.

Subsequent sections are concerned with the implementation of a complete QPSK transmission system with SCM. This paper focuses on the modulator distinctive features, including synchronization of the transmitted data to a prescaled version of the subcarrier signal and the application of the latter to provide a pilot tone for use in the system receiver. It also provides a detailed description of the specially designed PLL and symbol-timing circuitries, to down-convert the modulated signals and recover the transmitted symbols. The effects of jitter and intersymbol interference (ISI) on the observed eye patterns are investigated, following the transmission of a $675 \mathrm{Mb} / \mathrm{s}$ data signal over an optical link.

\section{NOVEL SUBCARRIER MODEM WITH QUADRATURE-PHASE MODULATION}

The experimental set-up is shown in Fig. 1. Its maximum specification in radio frequencies (RF) was dictated mainly by the radio frequency/local oscillator $(\mathrm{RF} / \mathrm{LO})$ operating range of the frequency mixers. This determined the range of subcarrier frequencies suitable to transmit information over the optical link. The information-bearing capacity of the system was defined primarily by the performance of the integrated components within the serial-to-parallel (S/P) and parallel-to-serial (P/S) converters.

Emitter coupled logic (ECL) level surface-mount logic devices were used to implement these converters, since they demonstrated the highest speed specifications commercially available at the time (up to $3 \mathrm{~Gb} / \mathrm{s}$ ). Also, operational amplifiers with signaling speeds up to approximately $1 \mathrm{~Gb} / \mathrm{s}$ were used to implement the unipolar-to-bipolar (U/B) converters [10]. Silicon Germanium (SiGe) components are commercially available today capable of processing several $\mathrm{Gb} / \mathrm{s}$.

Two orthogonal subcarriers at $2.7 \mathrm{GHz}$ were employed to transmit the information data. This frequency was chosen mainly for demonstration purposes, since a bandpass filter (BPF) and voltage-controlled oscillator (VCO) at $2.7 \mathrm{GHz}$ central frequency were available at the time. Succeeding work has demonstrated the transmission of $2.55 \mathrm{~Gb} / \mathrm{s}$ quadrature rates over a $5.1 \mathrm{GHz}$ subcarrier using the same setup, to efficiently use the optical bandwidth available [11].

\section{AnAlysis OF MODUlator Distinctive FEATURES}

The presented MODEM provides a novel configuration over conventional QPSK designs [12], since it employs distinctive circuit configurations, demonstrating error-free transmission and detection of high-speed signals with enhanced performance and less complexity. In that sense, a combination of prescalers, in conjunction with the pilot tone reinjection scheme and purpose-designed PLL provided simple CR and STR subsystems.

In the modulator, a pattern generator was set to produce unipolar, non-return-to-zero (NRZ) pseudorandom binary sequences (PRBSs) $2^{7}-1$ bits long, since NRZ coding is used particularly in short-link MMF applications where chromatic dispersion is not of concern. A standard 127-bit-long sequence length was chosen to reduce the processing time for BER calculations while providing sufficient power spectrum density to evaluate the CR and STR circuitries capability for symbol detection in white noise. PLL stable operation for data rates up to $2^{13}-1$ bits long was also demonstrated. A $12-\mathrm{GHz}$ BERT set was used to process a $675 \mathrm{Mb} / \mathrm{s}$ PRBS, to match the specifications of other components in the system. Two special features in the modulator were the external triggering of the pattern generator and the reinjection of the $2.7 \mathrm{GHz}$ subcarrier, at the point where the quadrature signals were combined, to provide a pilot tone for use at the system demodulator.

An external clock at $675 \mathrm{MHz}$, one-quarter the RF generator's subcarrier frequency, was applied to trigger the device by passing the subcarrier signal through a $9 \mathrm{GHz}$ divide-by-4 prescaler to produce a $675 \mathrm{Mb} / \mathrm{s}$ data sequence, within the processing limit of the MODEM. The purpose of this design was to synchronize or "lock" the transmitted sequence to the subcarrier frequency. In that sense, only a prescaler in the system demodulator was enough to extract the clock sequence required for symbol detection. This technique offered great simplicity, since a configuration of two identical prescalers prevented the requirement of a threshold detector, splitter, delay line, multiplier, and extra BPF, employed in conventional STR circuits [13]. Another feature of the design is the reduced sensitivity to additive white Gaussian noise (AWGN) and intermodulation products, especially in high frequencies, since the recovered clock was obtained from the extracted subcarrier with measured SNR of $30 \mathrm{~dB}$, through the use of the pilot tone and prescaler, and not from a highly attenuated, distorted, demodulated data signal, as is usually the case in conventional designs.

The second special feature is the reinjection of the subcarrier frequency at the point where the quadrature signals combine. The purpose of this scheme is to provide a pilot tone at 

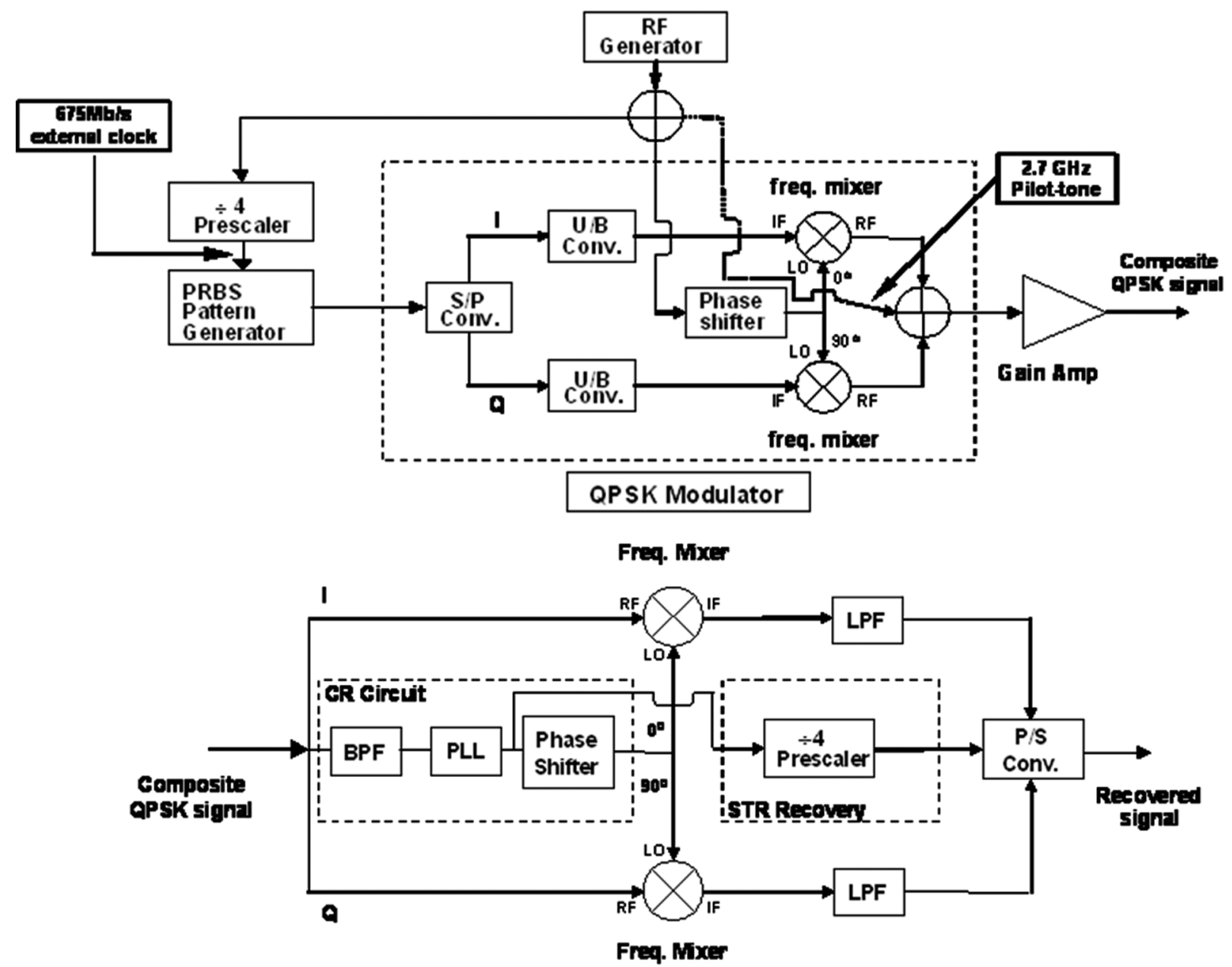

\section{QPSK Demodulator}

Fig. 1. Novel QPSK modulator-demodulator: IF up to $1 \mathrm{~Gb} / \mathrm{s}$, RF/LO up to $8 \mathrm{GHz}$.

the receiving end of the system, resulting in a simpler $\mathrm{CR}$, and therefore, cost-effective design that consists only of a BPF and a basic PLL, in contrast to "Costas" loops. The presence of the pilot tone in the detection of the transmitted symbols caused no degradation in the system's $10^{-9}$ BER performance, since it was shown that for a $0.07 \mathrm{~V} \mathrm{pk-pk}$ subcarrier, a maximum power penalty of $0.79 \mathrm{~dB}$ was incurred in the system SNR, compared with conventional binary designs.

The reinjected subcarrier provided a spectral line at the subcarrier frequency approximately $10 \mathrm{~dB}$ higher in power than the bandlimited spectrum at the output of the narrowband BPF. This was adequate for the PLL to acquire lock and extract the subcarrier signal with measured SNR of approximately $30 \mathrm{~dB}$. The constructed loop also demonstrated a phase lock between the transmitted and extracted subcarriers close to the theoretical $90^{\circ}$ with a measured phase margin of $85^{\circ}$ before losing lock, resulting in increased loop stability.

Apart from the synchronization and subcarrier reinjection schemes, the modulator employed S/P and U/B converters to split the incoming data sequence to in-phase (I) and quadrature-phase $(\mathrm{Q})$ data streams and convert them into bipolar signaling pulses, respectively. Conventionally, a $90^{\circ}$ phase shifter split the RF generator $2.7 \mathrm{GHz}$ subcarrier into I and Q signals, modulated by the bipolar data streams at the output of the U/B converters, into two frequency mixers.

\section{A. Pilot Tone Reinjection}

The bipolar streams were taken to the intermediate frequency (IF) ports of two identical frequency mixers for signal upconversion, as shown in Fig. 1. The RF generator output was split three ways to provide the pilot-tone reference and prescaler input signals, as well as the input to the phase shifter. The splitter port used to reinject the subcarrier frequency at the point where the quadrature signals combined, was heavily attenuated to provide the power combiner at the modulator output with a sinusoid at $2.7 \mathrm{GHz}$ around $0.07 \mathrm{~V}$ in amplitude. The output connections of the shifter were kept as short as possible and of the same length to keep their phase difference constant.

The composite QPSK signal was generated by combining the quadrature waveforms with the highly attenuated subcarrier sinusoid in a three-way $0^{\circ}$ combiner. Since the oscilloscope trace of the experimental QPSK waveform was very complex, to clearly display the contribution of the reinjected subcarrier in varying the amplitude of the composite QPSK signal, two 


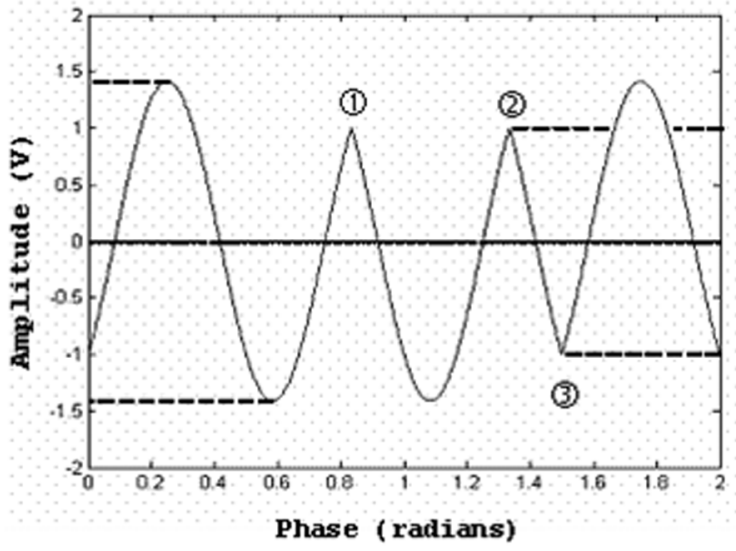

(a)

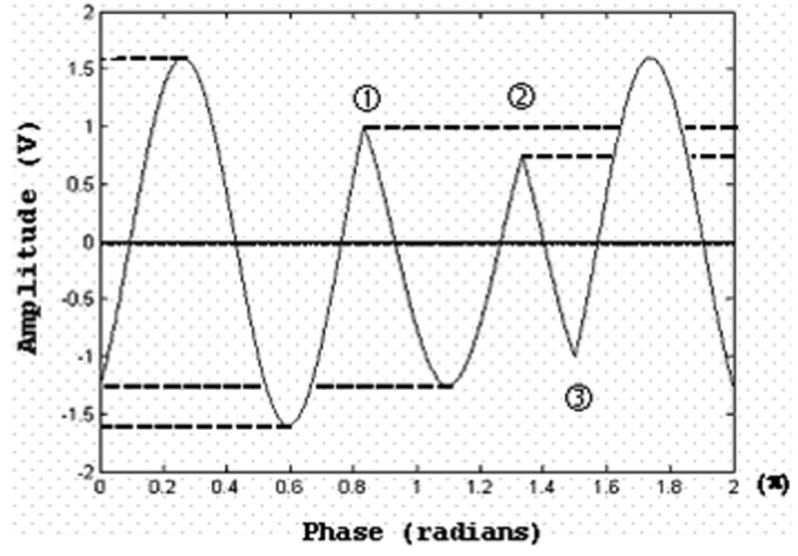

(b)

Fig. 2. Representative output of modulator subsystem. (a) Combined I and Q channels. (b) Effect of pilot tone reinjection at $I+Q$ waveform.

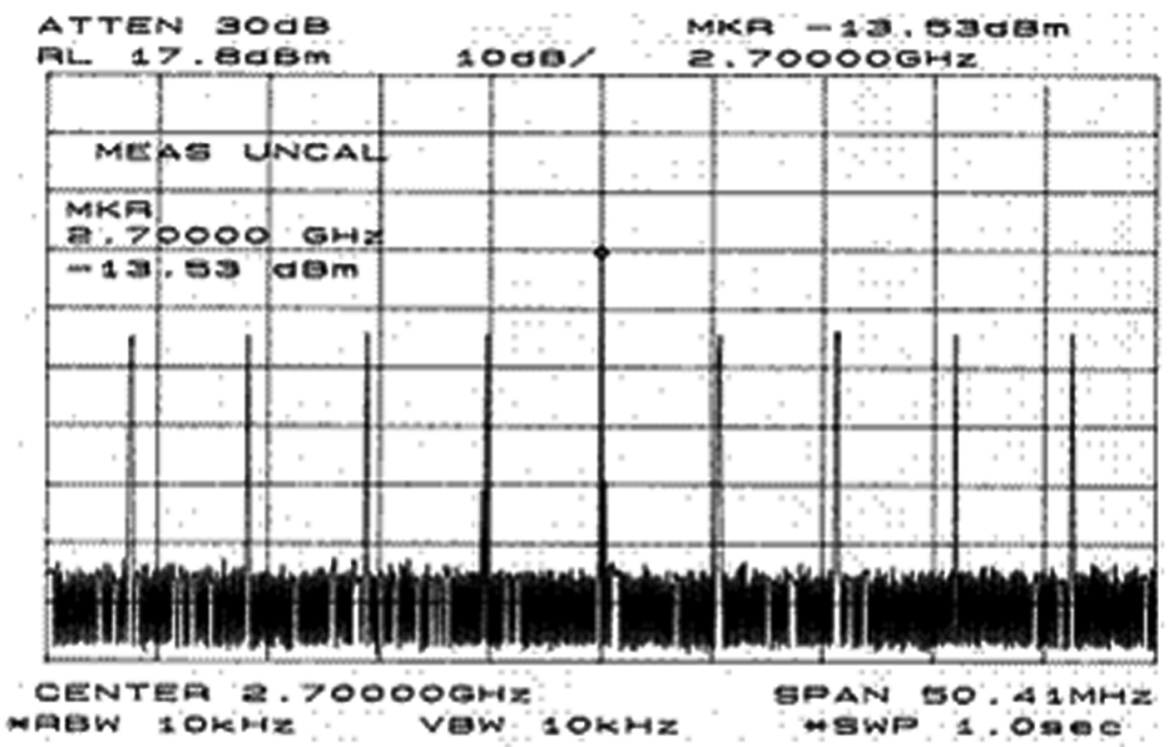

Fig. 3. Experimental output of modulator subsystem; displayed spectrum around the subcarrier frequency.

sinusoids in quadrature and a third of smaller amplitude were added in the MATLAB simulator, to represent the modulated waveforms and the reinjected signal, respectively. To be able to observe the modifications in the composite signal due to the pilot tone, a subcarrier signal frequency at $337.5 \mathrm{MHz}$, equal to the data rate of each modulating I and Q pulse streams, was inserted in the model instead of the eight times faster experimental $2.7 \mathrm{GHz}$ frequency.

The modulated I and Q waveforms exhibited a single and two successive symbol transitions, respectively, in order to investigate all possible combinations of phase changes. A pilot tone with an amplitude equal to a quarter of the magnitude of each quadrature channel was simulated to match the experimental pilot-tone amplitude and represent the imbalance superimposed in the composite QPSK signal. Waveforms in Fig. 2(a) and (b) display the combined signals with and without the reinjected subcarrier, respectively.

The phase change points 1, 2 and 3 in Fig. 2, corresponding to symbol transitions of the data stream, did not originate at zero amplitude of the composite waveform, since the combined quadrature channels did not experience phase inversion simultaneously at those instants due to the random nature of the data stream. The effect of the pilot tone on the $I+Q$ waveform was presented graphically by the amplitude variations in its response, shown in Fig. 2(b).

While in the absence of the reinjected signal the waveform displayed constant amplitude after changes in its phase, the composite quadrature plus subcarrier signal illustrated amplitude variations, either decreasing or increasing, according to the phase relation between the combined waveforms. The amplitude imbalance had the effect of producing a spectral line in the composite signal frequency response. This provides a pilot tone for use in the system.

In practice, the modulator output exhibited a multiamplitude signal with a spectral line at $2.7 \mathrm{GHz}$, which was approximately $10 \mathrm{~dB}$ higher than the highest power component in the transmitted signal spectrum. The spectrum of the experimental signal is shown in Fig. 3. 


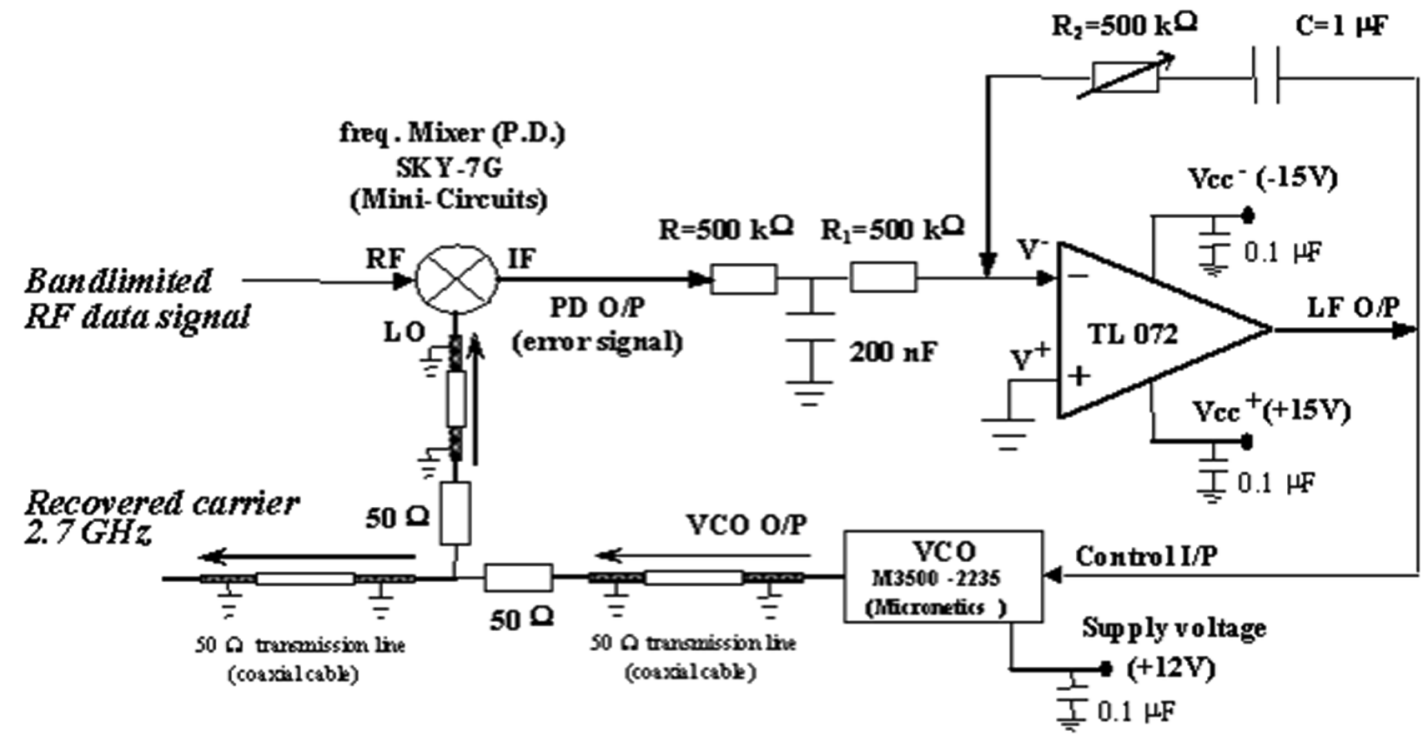

Fig. 4. Implemented PLL at $2.7 \mathrm{GHz}$.

\section{SySTEM DEMOdULATOR}

After broadband amplification, the composite QPSK waveform was applied to the demodulator subsystem for down-conversion and symbol detection. The main objective here was to accomplish a fully operational PLL at $2.7 \mathrm{GHz}$ to match system specifications. The STR circuit was implemented by passing the recovered subcarrier through a divide-by-4 prescaler, due to the prescaler locking of the transmitted data sequence to a quarter of the subcarrier frequency in the system modulator.

Down-conversion of the received quadrature signals and their translation to a serial data stream at twice the frequency were performed, respectively, by using two frequency mixers, with the same specifications as the ones used in the modulator, and a P/S converter comprised of the same components as its S/P counterpart, modified to perform P/S conversion.

\section{A. Pilot-Tone PLL For Subcarrier Extraction}

The requirement of tight synchronization between the transmitted and extracted subcarriers and clock sequences implies increased cost and complexity, by means of additional $\mathrm{Gb} / \mathrm{s}$ state-of-the-art components, but also improved system performance. The subcarrier extraction circuit is comprised of a 40 $\mathrm{MHz}$ bandwidth BPF centered at $2.7 \mathrm{GHz}$ and a basic PLL. The constructed PLL, shown in Fig. 4, employed a frequency mixer as a phase detector (PD), a pole-zero loop filter (LF) for increased stability, and a VCO. An acquisition circuit was used to help the loop acquire lock, since the initial difference between the subcarrier and VCO central frequencies was greater than the loop's pull-in range.

The performance evaluation of the CR subsystem was carried out by assuming narrowband AWGN at the loop input and phase noise due to the VCO operation. Both these noise sources can have a direct effect in the estimation of phase error if not treated carefully. Due to phase noise, the spectrum around the loop's operating frequency broadens, increasing the power of unwanted harmonics when combined with Gaussian noise in its input signal.

In practice, phase noise was brought to a minimum by selecting a VCO component with a low noise specification, and, equally important, by employing effective power supply bypassing and RF grounding when it was connected in the system. Extra consideration was taken to keep connections to the control input as short as possible and earth the unused pins and case ground at the same point. Coaxial cable transmission lines were used for the VCO output, which was passed through a power divider network to provide the PD input and PLL output.

The contribution of AWGN to the estimation of the loopphase error was determined by considering a linearized model. The demonstrated PLL was approximated by this model accurately, since the added pilot tone had the effect of increasing the PLL input signal power by providing a spectral component at the subcarrier frequency, while the employment of an active LF with narrow natural bandwidth provided a low-noise figure around the loop. Also, the narrowband BPF at the PLL input suppressed Gaussian noise to a great extent, since it supplied the loop with a 40-MHz bandwidth signal around the required $2.7 \mathrm{GHz}$ frequency, as shown in Figs. 3 and 7, displaying the signal spectrum before and after the application of the CR circuit.

To extract the subcarrier, the bandlimited QPSK signal and pilot tone were applied to the constructed loop input. The separation of the discrete spectral lines in the power spectrum of the input signal was determined by considering the period and symbol rate of the system information sequence [14]. For the $675 \mathrm{Mb} / \mathrm{s}, 127-$ bit-long PRBS used, this corresponded to a spectral line separation of $2.65 \mathrm{MHz}$. Therefore, the PLL input spectrum contained seven harmonics either side of the $2.7 \mathrm{GHz}$ subcarrier due to the BPF bandwidth.

After about three cycles, the transient phenomena in the loop had vanished, and the PLL got into lock in around $1.5 \mathrm{~s}$. The loop is expected to lock within a few milliseconds if the initial subcarrier and VCO central frequencies difference is smaller than 
the loop's pull-in range and an acquisition circuit is not required. The PLL input and the VCO output sinusoids displayed a fixed phase difference, while the error signal consisted of a DC waveform given by the loop filter (LF) gain factor $K_{\mathrm{LF}}$ multiplied with the sine of the fixed phase difference.

Tracking the subcarrier spectral line in a spectrum analyzer, displayed the $2.1 \mathrm{GHz} \mathrm{VCO}$ center frequency, moving under the control of the acquisition circuit towards the lock-in range of the loop. When inside the lock-in range, the feedback nature of the PLL forced the VCO frequency to lock, producing a spectral component at $2.7 \mathrm{GHz}$ with SNR of $30 \mathrm{~dB}$.

To define the open-loop gain expression, the gain of the LF, $K_{\mathrm{LF}}$, had to be determined. An active network with a negative feedback op-amp of gain factor 2 was employed for enhanced stability, since it was capable of tracking the changes in the PLL input signal phase by providing a high loop gain while, at the same time, being immune to bandlimited noise in the PLL input or phase noise in the VCO. Noise immunity was achieved by a narrow natural frequency design. As a result, the appropriate LF provided the PLL with the satisfactory phase margin defined next.

An advantage of this design, compared with passive filters, was its ability to provide a relatively low natural bandwidth, for the same loop gain requirement, by using resistors and capacitors with reasonable specifications [15]. Subsequently, it came down only to the appropriate selection of the filter components to reduce PLL phase error dramatically, which resulted in accurate tracking of the transmitted subcarrier characteristics. Also, the active filter configuration provided a wider lock-in range, allowing the designer to select the $\mathrm{VCO}$ with greater freedom.

\section{B. Evaluation of System Performance}

The PLL dynamic performance was determined by deriving its Bode diagram. The time constants $\tau_{1}, \tau_{2}$, and natural angular frequency $\omega_{n}$ of the LF are the actual parameters that controlled the PLL bandwidth and defined the achieved phase margin. To determine these parameters, the PD gain factor and VCO modulation sensitivity were considered first. Consequently, the loop filter and resistor-capacitor (RC) circuitry components, shown in Fig. 4, were selected according to the mixer and op-amp characteristics and the loop design requirements.

Since the detector output was terminated to $50 \Omega$ and the op-amp exhibited high input impedance, a resistance, matching the amplifier impedance, was required between the components to block the DC offset reflected back to the detector and also propagate the detector output through to the amplifier without attenuation. Also, time constant $\tau_{1}$ was expected to be as small as possible, but larger than $\tau_{2}$. For that to be the case, a small capacitor was required to provide negative feedback in the loop filter. By knowledge of the time constants and the PD and VCO gain factors the loop natural frequency, open-loop gain, and phase margin were specified.

The requirement of good stability states that if $\omega_{n} \tau_{2}>1$, the total phase shift around the loop falls between $-90^{\circ}$ and $-135^{\circ}$, allowing good phase margin. Also, the higher the PLL damping

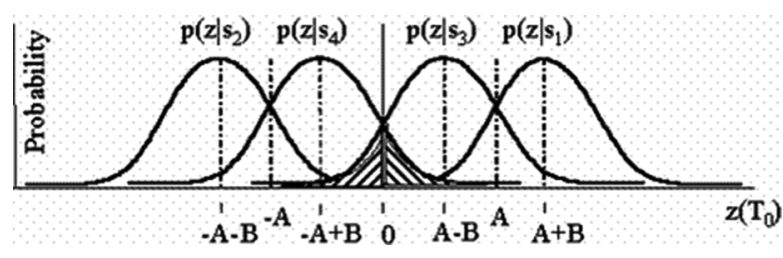

Fig. 5. Conditional pdfs in the transmission of bipolar binary pulses in the presence of the pilot tone.

factor, the better the phase margin. For a damping factor $\zeta$ of 0.707 , used in most applications, the loop stability requirement was achieved, since for the experimental PLL, $\omega_{n} \tau_{2}=8.8>$ 1. Loop stability was verified by calculating the phase shift at the frequency of unity open-loop gain. This corresponded to roughly $95^{\circ}$, well inside the required range, with a comfortable margin of $85^{\circ}$ indicating that even in the case of spurious time constants due to lowpass filtering at the PD output and VCO modulation, the phase margin was adequate for the loop to remain stable.

\section{Symbol Detection in the Presence of A Pilot Tone}

Since the I and Q signals are orthogonal BPSK waveforms, they can be studied independently. Fig. 5 demonstrates the decision signal space when the pilot tone is reinjected to either of the quadrature waveforms. Since the pilot tone has the effect of increasing or decreasing the QPSK waveform's amplitude by $B$, depending on the phase correlation of the signals at integer values of the symbol transitions, the decision signal space of the composite waveform will consist of four equally probable probability density functions (pdfs) corresponding to the four possible amplitude levels, in contrast to the two pdfs present in conventional binary systems.

The likelihoods of the four possible amplitude levels, shown in Fig. 5, are represented by $p\left(z \mid s_{1}\right), p\left(z \mid s_{2}\right), p\left(z \mid s_{3}\right)$, and $p\left(z \mid s_{4}\right)$. Parameters $z\left(T_{0}\right)$ and $\pm \mathrm{A}$ denote, respectively, the correlator output random variable, sampled at symbol intervals $T_{0}$, and the mean values of the conditional pdfs in the binary signal space. Assuming equally probable transmission of the four possible waveforms $s_{1}, \ldots, s_{4}$ and symmetry between their conditional pdfs $p\left(z \mid s_{1}\right), p\left(z \mid s_{2}\right), p\left(z \mid s_{3}\right)$, and $p\left(z \mid s_{4}\right)$, $P_{E}$ in the presence of the pilot tone will be expressed by

$$
\begin{aligned}
P_{E}= & \frac{1}{4} \int_{-\infty}^{0} p\left(z \mid s_{1}\right) d z+\frac{1}{4} \int_{0}^{+\infty} p\left(z \mid s_{2}\right) d z \\
& +\frac{1}{4} \int_{-\infty}^{0} p\left(z \mid s_{3}\right) d z+\frac{1}{4} \int_{0}^{+\infty} p\left(z \mid s_{4}\right) d z .
\end{aligned}
$$

Considering the signal space diagram of Fig. 5, $P_{E}$ results in

$$
\begin{aligned}
P_{E} & =\frac{1}{4}\left(\int_{-\infty}^{0} p\left(z \mid s_{3}\right) d z+\frac{1}{4} \int_{0}^{+\infty} p\left(z \mid s_{4}\right) d z\right) \\
& =\frac{1}{4} \times 2 \int_{0}^{-\infty} p\left(z \mid s_{4}\right) d z+\frac{1}{2} \int_{0}^{+\infty} p\left(z \mid s_{4}\right) d z
\end{aligned}
$$




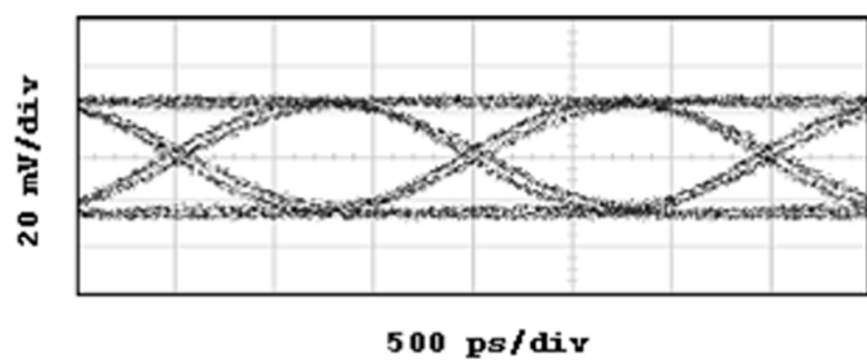

Fig. 6. Recovered eye diagram at the output of the $675 \mathrm{Mb} / \mathrm{s}$ QPSK MODEM.

Such a simplification is valid, since the contribution of the tail areas underneath the pdfs with mean values $+A-B$ and $-A-B$, to the overall system error is much smaller compared with the corresponding tail areas underneath the pdfs with mean values $-A+B$ and $-A+B$. Therefore, the first two parameters in (1) can be neglected. To determine the effect of the pilot tone on the system performance, a $P_{E}$ of $10^{-9}$ is assumed for the conventional case. From the standard error function graph and (2), the ratio of the signal amplitude to the noise standard deviation for the cases without and with pilot tone are expressed by

$$
\begin{aligned}
\frac{-A}{\sigma} & =6.025 \\
\frac{-A+B}{\sigma} & =5.925 .
\end{aligned}
$$

Division of the equations in (3) showed that $B=0.017 A$. For $A=0.4 \mathrm{~V}$, as was the case in practice, this resulted in a pilot tone mean value of $B=0.007 \mathrm{~V}$. This value corresponds to the optimum pilot-tone level that demonstrates no apparent degradation in the system's performance. In practice, a pilot-tone level of $0.035 \mathrm{~V}$ was used, which resulted in a maximum power penalty of $0.79 \mathrm{~dB}$ in the received optical signal SNR, compared with the conventional system. Such a power penalty was acceptable in order to maintain system performance (BER of $10^{-9}$ ), while the implemented PLL provided simple subcarrier extraction for RF frequencies in the range of $2.5-2.8 \mathrm{GHz}$.

\section{Transmission of the Quadrature Data OVER Fiber}

The modulator QPSK output was transmitted for demonstration purposes over $25 \mathrm{~km}$ of a standard SMF link to offer a useful insight into its subcarrier-extraction performance in the case of low SNR. The QPSK signal was used to externally trigger a laser source through a Mach-Zehnder (MZ) modulator. The optical signal, after polarization control, was connected through a SMF patchcord to the SMF transmission reel, followed by a SMF photodiode.

Initially, the MODEM performance was investigated in a back-to-back configuration, by determining the effect of data transition and clock jitter on the quality of the recovered eye diagram, shown in Fig. 6. The recovered pattern demonstrated approximately $8 \%$ and $30 \%$ of the symbol duration, occupied by clock and data-transient jitter, respectively. Also the amount of eye closure due to ISI corresponded to around $-1.5 \mathrm{~dB}$ peak degradation, demonstrating a highly efficient MODEM design. The system performance is expected to be higher if the implemented subsystems are properly implemented on printed circuit boards.

The $675 \mathrm{Mb} / \mathrm{s}$ data signal was then transmitted over $25 \mathrm{~km}$ of SMF. The output of the photodiode was amplified by $23 \mathrm{~dB}$ and applied to the splitter to provide the input to the BPF and PLL. Fig. 7 shows the recovered subcarrier signal with measured SNR of around $30 \mathrm{~dB}$.

In the course of constant operation for a period of two days, the PLL displayed remarkable stability to input power variations that could arise due to temperature dependence of various components, such as lasers, optical modulator, and electrical amplifier, and spurious phenomena that could have taken place due to interference with signals from running experiments in the laboratory. It was also very efficient in acquiring lock once working within specifications. Power budget analysis demonstrated consistent PLL operation for input signal powers as low as $-10 \mathrm{dBm}$, due to individual loop component requirements and potential power leakage in the design. Compact PCB designs are expected to have superior power budget performance.

Once again the displayed eye diagram and BER measurements demonstrated error-free transmission of $10^{-9}$ at $675 \mathrm{Mb} / \mathrm{s}$, at $0 \mathrm{dBm}$ optical power. An extra degradation of approximately $1 \mathrm{~dB}$ was incurred by the signal traveling down the optical link, which resulted in a total eye closure of around $2.5 \mathrm{~dB}$. The recovered eye pattern is shown in Fig. 8 .

\section{ENHANCED System PERFORMANCE}

The optical system for which the MODEM was developed was capable of processing data rates around $6 \mathrm{~Gb} / \mathrm{s}$ and subcarrier frequencies in the range of 4-6 GHz. Although the setup of Fig. 1 was capable of accommodating RF signals up to $8 \mathrm{GHz}$, it was restricted to IF rates up to $1 \mathrm{~Gb} / \mathrm{s}$, due to limitations in the ECL-level logic components within the S/P and P/S converters. Enhancing the QPSK setup performance required initially omitting the system converters and introducing an equivalent modulation scheme. This was achieved by considering the concept of an I and Q MODEM.

The reconfigured experimental setup employed a $5.1 \mathrm{GHz}$ subcarrier, used to trigger the generation of $2.5 \mathrm{~Gb} / \mathrm{s}$ data sequences through a divide-by- 2 prescaler. The setup exploited the same properties with those described before and the same PLL design. The VCO was the only device replaced with its replacement operating at $5.1 \mathrm{GHz}$. The enhanced modem demonstrated the transmission of $5.1 \mathrm{~Gb} / \mathrm{s}$ aggregate data rates over $3 \mathrm{~km}$ of standard MMF reels. Somewhat 10 times more data over 10 times the distance of conventional MMF links [11].

The comprehensive combination of the modem with a highly dense WDM scheme demonstrated a potential throughput of $1.02 \mathrm{~Tb} / \mathrm{s}$ over $3 \mathrm{~km}$ of installed-base MMF, featuring a world record transmission over MMF to date [16].

\section{CONCLUSION}

We described the implementation of a novel SCM MODEM capable of accommodating aggregate data rates up to $1 \mathrm{~Gb} / \mathrm{s}$ and 


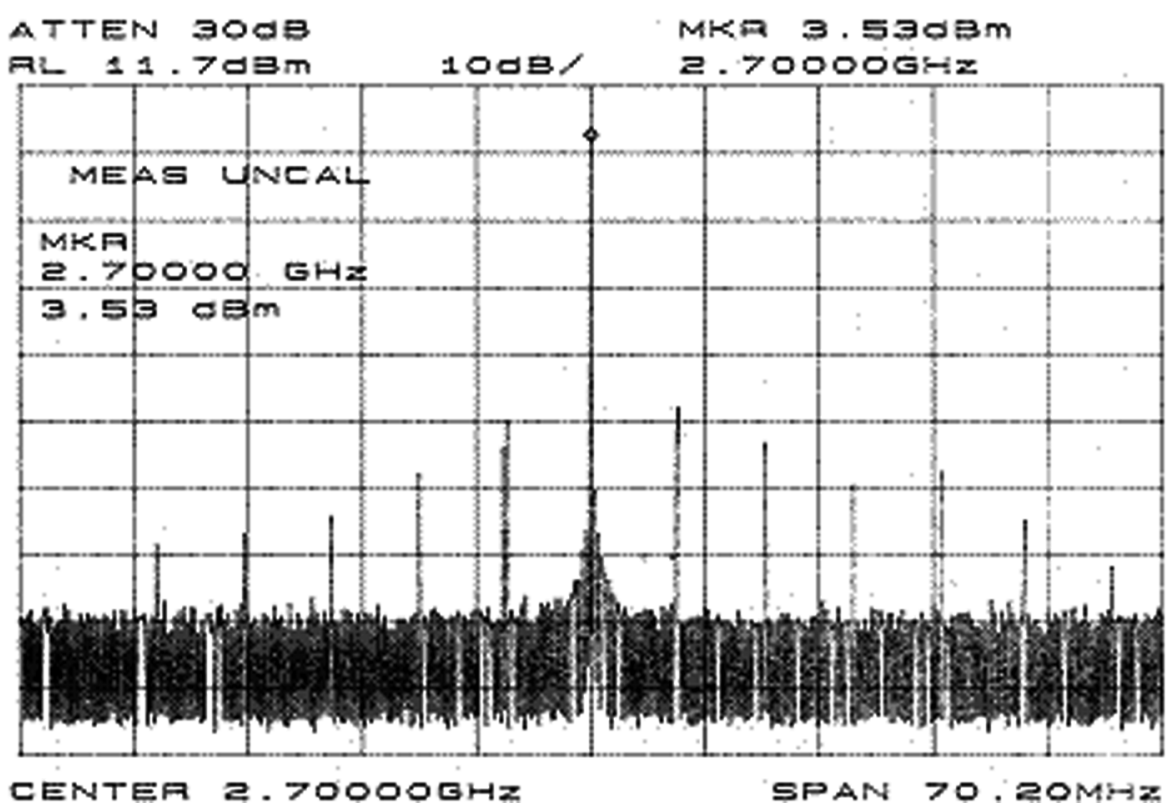

Fig. 7. Spectrum of recovered subcarrier signal at $2.7 \mathrm{GHz}$.

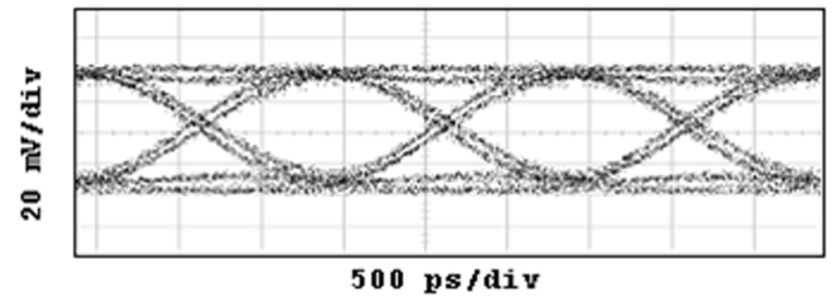

Fig. 8. Demonstrated eye diagram after transmission of the $675 \mathrm{Mb} / \mathrm{s}$ QPSK signal over $25 \mathrm{~km}$ of a standard SMF link.

subcarrier frequencies in the range of DC to $8 \mathrm{GHz}$. The setup demonstrated the transmission of a $675 \mathrm{Mb} / \mathrm{s}, 2^{7}-1$ PRBS in a QPSK format, where the I and Q symbol streams modulated the phase of the orthogonal components of a subcarrier frequency at $2.7 \mathrm{GHz}$.

Two distinctive features in the system modulator were the synchronization of the transmitted data sequences to one-quarter of the subcarrier frequency, through a prescaler and the reinjection of the unmodulated subcarrier to the point where the quadrature signals combined. In effect, the received QPSK signal employed a spectral line at $2.7 \mathrm{GHz}$, requiring just a basic PLL design to track and extract the subcarrier signal and a further divide-by- 4 prescaler to provide the time reference for symbol detection.

As a result, the implemented system provided much simpler, and hence, cost-effective CR and STR circuits than conventional designs at high frequencies, with no apparent degradation in the system's performance due to the presence of the pilot tone at the QPSK signal spectrum. The performance evaluation of the experimental setup, demonstrated satisfactory system behavior to data transition and clock jitter, as well as ISI effects. The specific design offered the basis of an enhanced system with even higher data-processing capabilities, used to transmit the highest information rate over MMF to date.

\section{REFERENCES}

[1] The Gigagit Ethernet Standard, IEEE 802.3z-1998, 1998.

[2] T. Kanada, "Evaluation of modal noise in multimode fiber-optic systems," J. Lightw. Technol., vol. LT-2, no. 1, pp. 11-18, Jan., 1984.

[3] M. Webster, A. B. Massara, I. H. White, and R. V. Penty, "10 Gb/s transmission over $300 \mathrm{~m}$ of standard multimode fiber using multilevel coding and 2-channel WDM,' in Proc. Conf. Lasers Electro-Opt., 2000, pp. 94-95.

[4] T. E. Darcie, "Subcarrier multiplexing for multiple-access lightwave networks," J. Lightw. Technol., vol. LT-5, no. 8, pp. 1103-1110, Aug. 1987.

[5] L. Raddatz et al., "High bandwidth data transmission in multimode fiber links using subcarrier multiplexing with vertical cavity surface emitting lasers," Electron. Lett., vol. 34, pp. 686-688, 1998.

[6] P. Kourtessis et al., "The applications of SCM in optical data communications," in Proc. Int. Topical Meet. Microw. Photon., 2002, pp. 363-366.

[7] T. E. Darcie, "Subcarrier multiplexing for lightwave networks and video distribution systems," IEEE J. Sel. Areas Commun., vol. 8, no. 7, pp. 1240-1248, Sep., 1990.

[8] L. Raddatz et al., "High data rate transmission of subcarrier multiplexed signals using vertical cavity surface emitting lasers and multimode fiber," in Proc. ECOC, Edinburgh, U.K., 1997, vol. 3, pp. 231-234.

[9] P. M. Hill and R. Olshansky, "Multigigabit subcarrier multiplexed coherent lightwave system," IEEE J. Lightw. Technol., vol. 10, no. 11, pp. 1656-1664, Nov. 1992.

[10] J. Goldie, "LVDS, CML, ECL-differential interfaces with odd voltages," 2003 [Online]. Available: PlanetAnalog.com

[11] P. Kourtessis et al., "A complete $5 \mathrm{~Gb} / \mathrm{s}$ throughput quadrature subcarrier system featuring zero-latency carrier and data synchronization," in Proc. OFC, 2002, Paper TuV1.

[12] H. Aghvami, "Digital modulation techniques for mobile and personal communication systems," Electron. Commun. Eng. J., vol. 5, no. 13, pp. 125-132, 1993.

[13] E. A. Lee and D. G. Messerschmitt, Digital Communication. Boston: Kluwer, 1988.

[14] A. Blanchard, Phase Locked Loops: Application to Coherent Receiver Design. New York: Wiley, 1976.

[15] A. J. Viterbi, Principles of Coherent Communication. New York: McGraw-Hill, 1966.

[16] P. Kourtessis et al., "Ultra-high capacity transmission over $3 \mathrm{~km}$ of legacy $50 \mu \mathrm{m}$-multimode-fiber using C-band HDWDM and quadrature-subcarrier multiplexing," in Proc. ECOC, Copenhagen, Denmark, 2002, Paper 8.2.2. 


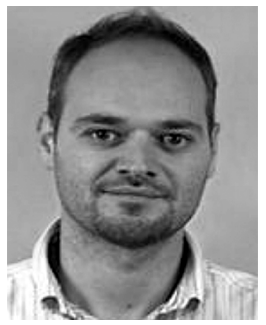

Pandelis Kourtessis (M'03) was born in Athens, Greece, in 1974. After receiving an honours B.Eng. degree in electrical and electronic engineering in 1997 from City University, London, U.K., he received the M.Sc. degree in telecommunications and information systems from the University of Essex, Colchester, U.K.

In 1998, he was offered a Ph.D. studentship from the Optical Systems Research Group of the University of Essex to work on a project funded by the U.K.'s Engineering and Physical Sciences Research Council. His thesis examined the transfer of data over installed-base multimode fiber links by combining spectrally efficient RF modulation techniques with a highly dense wavelength-division multiplexing scheme to demonstrate multi-gigabit/s LAN and campus area networks. In the course of his research, he was also employed as a Graduate Teaching Assistant. In 2003, he joined University of Hertfordshire, Herts, U.K. Currently a Senior Lecturer and member of the Optical Networks Group, his research focuses on physical layer architectures for integrating passive optical networks and bandwidth-allocation algorithms for the dynamic assignment of network traffic in the access. Recently, he has developed an interest in the interworking of wireless and optical technologies for the provision of multimedia services in future access/metro networks.

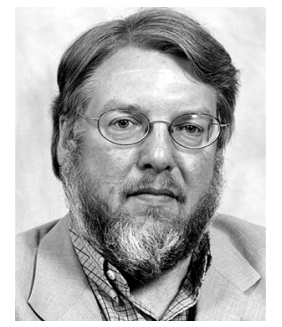

Stuart D. Walker was born in Dover, U.K., in 1952. $\mathrm{He}$ received the B.Sc. (Hons) degree in physics from Manchester University, Manchester, U.K., in 1973, and the M.Sc. degree in telecommunications systems and the Ph.D. degree in electronics from Essex University, Essex, U.K., in 1975 and 1981, respectively.

After a period of postdoctoral work at Essex University, he joined the then British Telecom (BTPlc) Research Laboratories, Ipswich, U.K., in 1982. Initially, he was concerned with regenerator design issues in submarine optical transmission systems. While at BT Plc, he was jointly responsible (with Prof. P. Cochrane) for pioneering the unrepeatered-transmission-system concept. In 1987, he was promoted to head the transatlantic link-repeater group, where he supervised the design and fabrication of high-reliability integrated circuits. In 1988, he became a Senior Lecturer at Essex University. There, his research interests included fiber-polarization studies and novel optoelectronic-device configurations. $\mathrm{He}$ then developed an interest in access-network design and construction, where he formed a specialist research group. Subsequently, he undertook an extensive series of optical-device studies in collaboration with Fujitsu Laboratories of Europe, Ltd. In 2003, he was promoted to Reader, and to Full Professor in 2004. 